\title{
USABILITY EVALUATION OF THE MOBILE APPLICATION FOR THE VIRTUAL MOBILITY LEARNING HUB
}

\author{
Diana Andone, Silviu Vert, Radu Vasiu, Oana Rotaru, Vlad Mihaescu, Daniela Stoica, \\ Victor Holotescu, Politehnica University Timisoara, Romania
}

\begin{abstract}
The use of MOOCs (Massive Online Open Courses) has become very popular in the past few years. Such courses can teach a large variety of skills, including the basics of virtual mobilities, both for students and teachers. The Open VMLH (Virtual Mobility Learning Hub) is an innovative multilingual environment which was created as part of the Erasmus+ Open Virtual Mobility project, with the purpose to promote collaborative learning, social connectivism and networking as an instructional method, OERs as the main content, and open digital credentials. In this paper, we will present the usability evaluation of the Open VMLH mobile application, a mix of heuristic and cognitive evaluation methods. Some of the issues discovered are related to the general interface and functionality of the mobile application, while others are related to the structure of the courses and the implementation of the content, but just a couple of them are critical for the user experience.
\end{abstract}

Keywords: Usability Evaluation; Human Computer Interaction; User Experience; User Testing; Remote Testing; Focus Group; User Observation; Expert Reviews; Learning Management System, Massive Open Online Course; Virtual Mobility.

\section{Introduction}

In the last years, the use of Massive Open Online Courses (MOOCs) has become a very popular way to deepen one's knowledge in a variety of fields. According to (Lee et al., 2018), a MOOC is practically an online learning platform with a large number of students. Going in the details of the term, a MOOC is addressed to a massive number of people, it is open to anyone, it is hosted online, and it offers educational resources that are accessible to anyone.

According to (Johansson \& Frolov, 2014), an even more extended definition of a MOOC includes the following aspects: an online course that doesn't need physical presence; an 
available and accessible course for everyone, from anywhere; the course is self-directed, self-paced or time limited, having a start and an end date; it consists of video lectures and/or readings, examinations in the form of assignments, exams, experiments; it supports interactivity between the participants and the tutors through online forums or other social media platforms; its content meets high academic standards; and it supports the creation of educational communities.

An example of MOOC platform is the Open VMLH (Virtual Mobility Learning Hub), an innovative multilingual environment which was created as part of the Open Virtual Mobility, a European-funded project, with the purpose to promote collaborative learning, social connectivism and networking as an instructional method, OERs (Open Educational Resources) as the main content and open digital credentials (Andone et al., 2020). The Virtual Mobility Learning Hub is available both on website and through a mobile application.

Following our research in (Andone et al., 2020), where the authors of this paper presented the usability evaluation for Open VMLH website, here we will present the usability evaluation of the Open VMLH mobile application.

The usability evaluation was organized entirely remotely, due to COVID-19 restrictions, by the authors and a group of students in the Multimedia Technologies master's degree program at the Politehnica University of Timişoara (Romania).

Usability is defined as a qualitative assessment of the user interface in a product, such as a website or a mobile application, in addition to its purpose that allows users to achieve specific goals (Johansson \& Frolov, 2014).

In this context, small screen size, limited interaction model, limited processing and storage space are aspects that make the mobile usability an important factor in mobile application development (Azzahra et al., 2015). However, users prefer mobile apps that are easy to learn, take less time when completing a task and, mobile apps with a dynamic interface (Nayebi et al., 2012).

\section{Related work}

Researchers from the e-Learning team of the Faculty of Computer Science, University of Indonesia, present in (Banimahendra \& Santoso, 2018) the implementation and the evaluation of a LMS mobile application named SCeLE (Student Centred e-Learning Environment). The usability evaluation of SCeLE involved the user observation session with 14 participants and the questionnaire method using the SUS questionnaire, with 107 respondents. Although the final results were good, the authors came up with the conclusion that some parts of the application must be improved, such as the login process, 
the order of some parts of the content, the search features of the forum or the notification features.

The authors in (Pensabe-Rodriguez et al., 2020) describe the evaluation of a Context-Aware Mobile Learning System (CAMLS) with six professors and forty-eight high school students. The evaluation, with was done as a field study in a real environment, showed $82.4 \%$ acceptance rate by both professors and high school students.

A systematic literature review was performed by the authors of (Kumar \& Mohite, 2018) in the field of usability testing of mobile learning applications. The authors provide a valuable insight into the methods, the gaps and the future research directions in the field.

\section{Usability evaluation of the open VMLH mobile app}

The VMLH mobile app, based on the Moodle open source app, is available for both Android (https://play.google.com/store/apps/details?id=eu.openvirtualmobility.hub) and iOS (https://apps.apple.com/app/id1481003592). The app features 8 mini MOOCs, each composed of 3 courses for Foundation Level, Intermediate Level and Advanced Level. The courses which were part of the app and evaluated are Media and Digital Literacy, Intercultural skills, Autonomy-driven Learning, Active Self-regulated Learning, Collaborative Learning, Networked Learning, Open mindedness, and Open Education and Virtual Mobility. Figure 1 shows the homepage of the MOOCs in the app.

\section{Methodology}

The evaluation of the OpenVM mobile application consisted in applying a mix of testing methods to identify usability issues in the app (and suggest recommendations to fix them).

The usability evaluation was designed around two main research questions:

- Q1: What are the experiences that real students and teachers might have as learners in the VMLH mobile app?

- Q2: Is the VMLH app error-free and ready to become available to the final users?

The entire usability testing process took place in May 2020, with the evaluation being realized by a team of 3 students, coordinated by the authors of this paper, as a group project for the Usability and Interactivity course which is taught in the Multimedia Technologies master's degree program at the Politehnica University of Timișoara (Romania).

The authors chose 3 evaluation methods to maximize the qualitative data which was to be gathered. These are the user observation method, the focus group, and the expert review method. 


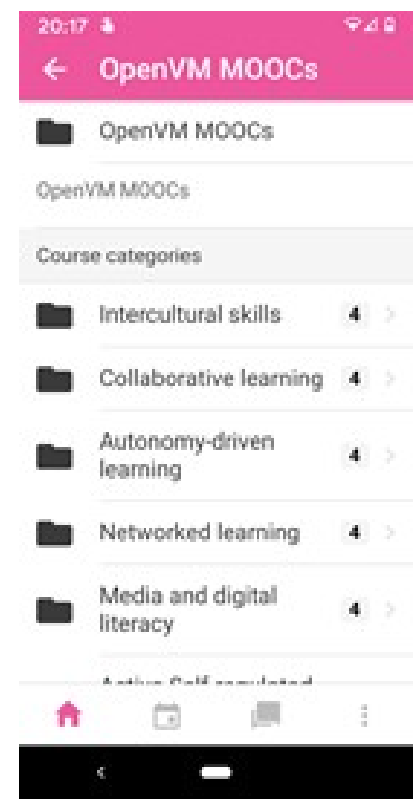

Figure 1. The home screen of the OpenVM mini MOOCs

In total, 12 participants took place in the evaluation: 9 university students and professors (as per the personas identified initially) and 3 mobile application experts.

Due to COVID-19 restrictions, the evaluation had to be realized completely remotely, using Microsoft Teams.

Each of the evaluation methods is described in the following subsections.

The User Observation Sessions

Because the mobile application is intended to be used both by students and professors, for the user observation method, the group of participants was composed by 3 students and 1 university professor.

Before the session, they had to fill in a prequestionnaire to give the authors a better view regarding their skills in using mobile applications and MOOCs. One of the participants had experienced a virtual mobility before.

The tasks they had to complete were designed to evaluate both the interface of the mobile application and the implementation of the MOOCs in the app.

Most of the tasks were completed with few errors during the process, however, the participants encountered some issues that affect the entire experience of using the application. The issues were related to the login process, the implementation of the multimedia elements (some of the videos did not work properly), the navigation buttons (some of them were not suggestive) and the content (which was not fully translated in all the app's official languages). 
At the end of the session, each participant had to complete a postquestionnaire to give more information about their overall experience within the application. In general, the participants said that their experience was pleasant, besides the small issues that they have encountered. They would use it again because it can help them become more productive.

\section{The Focus Group Session}

Five participants, university professors and students, were engaged in a focus group to give feedback about the OpenVM mobile application. As the others, the session was organized remotely, through Microsoft Teams.

Before the session started, all the participants completed the prequestionnaire to help the authors to create a more complex profile of the users.

At the first glance, they considered that the design of the application is appropriate, and they enjoyed the experience.

Walking through the mobile application they discovered that the video tutorial is not integrated properly and that on the main page they could not find any information regarding the purpose of the application. They also considered that the support sections need to be improved - this it would really help create a better experience for the future users.

The navigation was considered quite easy and intuitive, with few exceptions related to some parts of the menu. Also, they considered that the log out section is not intuitive at all and they struggled to find it.

Another thing that the participants appreciated was the integration of the interactive calendar functionality, but they stated that they were a bit confused because they did not figure out exactly what kind of events could be programmed using the calendar.

They were also a bit confused by the fact that the application contains 2 different sections where they could visualize their grades.

They graded the app as 7.5/10 and they also stated that they would recommend it to a friend.

\section{The Expert Review Sessions}

For this evaluation method, 3 mobile applications specialists were asked to walkthrough the mobile application and give feedback and recommendations to the usability engineers (Figure 2). 


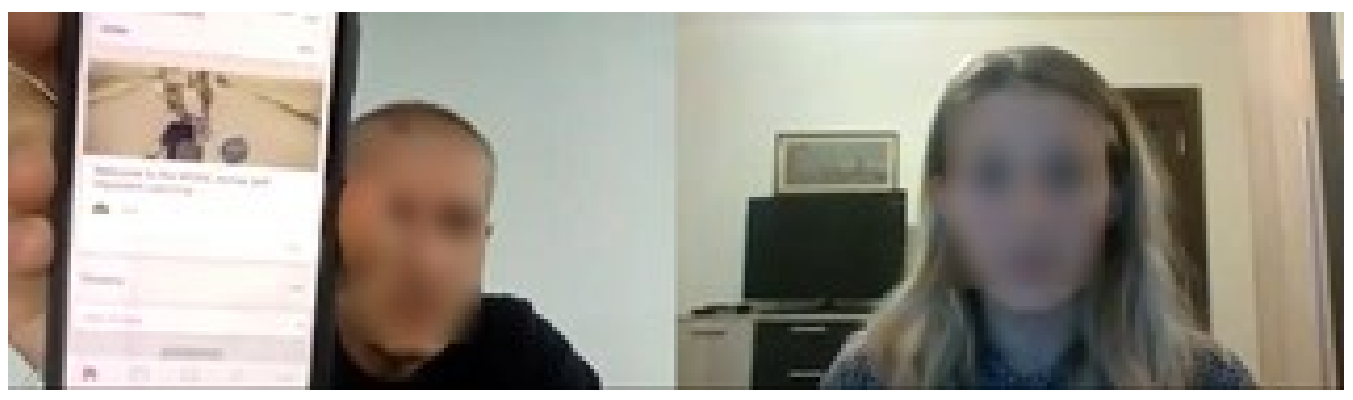

Figure 2. Screenshot from the one of the expert review sessions

This method was used because it can highlight many important issues in a short period of time and because, considering the experts' point of view, the issues discovered can be prioritized easily.

Some of their observations were related to the defective login process, the lack of the progress bar when downloading courses for offline usage, the missing support information regarding the use of the application, the grading process, and the matching between tags. Also, they noticed that the video tutorial does not have a preview image, so the users believe that it does not work.

Another issue was that some buttons, for example the refresh button, are not suggestive. The fact that the tutors' contact details are not displayed in each course was considered a usability issue.

\section{Results and recommendations}

After the authors analysed all the feedback obtained through the 3 evaluation methods, they made a classification of the issues identified.

Jakob Nielsen proposed a four-step scale to rate the severity of usability problems, as it follows (Nielsen, 2006): 0 - The problem is not a usability issue, 1 - Cosmetic problem only, 2 - Minor usability problem, 3 - Major usability problem, 4 - Usability catastrophe.

In the following part, the authors list the most frequent problems that have been reported, together with their severity rating (in parentheses).

Issues related to the mobile app's interface and general functionality:

- The login/sign up process is defective because even if the users can use multiple ways to sign up, only the Google login works properly (3).

- Some buttons' icons are not intuitive (e.g. log out button) (3).

- Integration of multimedia elements is defective (3).

- Search field is confusing (2).

- Help section lacks useful information (3). 
- The "after sign-up" form is not easily visible, so the participants did not complete it (3).

- "Forum" section redirects the user to the site announcements section (2).

- Feedback sections redirects the user to the sign-up form (3).

Issues related to the courses' structure and the implementation of the content in the mobile platform:

- Unavailable courses were not highlighted in any way, a fact that confused the users when browsing the courses (2).

- Some course sections were empty (2).

- Enrolment process is taking too long (2).

- "Download course" function is not well explained, and it created confusion among the participants (1).

- $\quad$ Not all the courses have a suggestive general description (2).

A list of recommendations was obtained from the participants after the sessions:

- The main page should contain more helpful information about the application and the courses.

- The participants would prefer the possibility to adapt the colours of the applications using the day-night view.

- The calendar section needs to be improved and to have support information attached.

- The videos' integration needs to be improved.

- The colours should be updated to point out the importance of different key sections and functions.

\section{Conclusions}

Nowadays Higher Education is transforming, as today most of the students are also working adults. Considering this aspect, online learning platforms, including the mobile learning environments, are flourishing.

These difficult pandemic times empower the idea that mobilities like Erasmus+ should be also available online. In this respect, mobile applications such as the "Open Virtual Mobility Learning Hub" mobile app are an important and even mandatory resources for both professors and students.

The evaluation of the OpenVM mobile application was organized remotely and a mix of both heuristic and cognitive methods were used to obtain the best qualitative results. The methods used were the user observation method, the focus group, and the expert review method. 
The usability evaluation allowed us to answer the two main research questions as follows:

- A1: According to the participants, the overall experience was a good one and they really appreciated the opportunity to join a virtual mobility online, without having to go to another country. However, some specific usability problems related to the mobile app's interface and general functionality can seriously hinder their learning process.

- A2: The VMLH mobile app is not error-free and improvements need to be done before being made available to the final users. Most of the issues are in the areas of support information, navigation buttons and courses' content.

As a side note, this usability evaluation also contributes as a valuable example to the body of knowledge of implementing real-life cases of lab work with students.

As future work, we plan to solve the issues discovered and to add more functionalities to the mobile application, and to organize another usability evaluation to test the improvements.

\section{References}

Andone, D., Vert, S., Mihaescu, V., Stoica, D., \& Ternauciuc, A. (2020). Evaluation of the Virtual Mobility Learning Hub. In P. Zaphiris \& A. Ioannou (Eds.), Learning and Collaboration Technologies. Designing, Developing and Deploying Learning Experiences (pp. 20-33). Springer International Publishing. https://doi.org/10.1007/978-3-03050513-4_2

Az-zahra, H. M., Pinandito, A., \& Tolle, H. (2015). Usability evaluation of mobile application in culinary recommendation system. Paper presented at the 2015 IEEE Asia Pacific Conference on Wireless and Mobile (APWiMob). https://doi.org/10.1109/APWIMOB.2015.7374938

Banimahendra, R. D., \& Santoso, H. B. (2018). Implementation and evaluation of LMS mobile application: Scele mobile based on user-centered design. Journal of Physics: Conference Series, 978, 012024. https://doi.org/10.1088/1742-6596/978/1/012024

Johansson, S., \& Frolov, I. (2014). An Adaptable Usability Checklist for MOOCs: A usability evaluation instrument for Massive Open Online Courses. Umeå University, Department of Informatics.

Kumar, B. A., \& Mohite, P. (2018). Usability of mobile learning applications: A systematic literature review. Journal of Computers in Education, 5(1), 1-17. https://doi.org/10.1007/s40692-017-0093-6 
Lee, J., Hong, A., \& Hwang, J. (2018, June). A Review of Massive Open Online Courses: MOOC's Approach to Bridge the Digital Divide. Paper presented at the $22^{\text {nd }}$ Biennial Conference of the International Telecommunications Society (ITS): Beyond the Boundaries: Challenges for Business, Policy and Society, Seoul, Korea, 24th-27th June, 2018. Retrieved from http://hdl.handle.net/10419/190394

Nayebi, F., Desharnais, J.-M., \& Abran, A. (2012). The state of the art of mobile application usability evaluation. Proceedings of the $201225^{\text {th }}$ IEEE Canadian Conference on Electrical and Computer Engineering (CCECE), 1-4. https://doi.org/10.1109/CCECE.2012.6334930

Nielsen, J. (2006). Severity Ratings for Usability Problems.

Pensabe-Rodriguez, A., Lopez-Dominguez, E., Hernandez-Velazquez, Y., DominguezIsidro, S., \& De-la-Calleja, J. (2020). Context-aware mobile learning system: Usability assessment based on a field study. Telematics and Informatics, 48, 101346. https://doi.org/10.1016/j.tele.2020.101346

\section{Acknowledgments}

The current study has been carried out in the framework of the Erasmus+ Project Open Virtual Mobility, key action Cooperation for Innovation and the Exchange of Good Practices, Strategic Partnerships for higher education, (partially) founded by the European Union, Project Number 20171-DE01-KA203-003494. We would like to thank students Daniela Petcu, Andra Maskovszky and Marian Belean for their outstanding attention to details in running this usability evaluation. 